\title{
Thermal neutron capture cross section of the radioactive isotope ${ }^{60} \mathrm{Fe}$
}

T. Heftrich,,${ }^{1, *}$ M. Bichler, ${ }^{2}$ R. Dressler, ${ }^{3}$ K. Eberhardt, ${ }^{4}$ A. Endres, ${ }^{1}$ J. Glorius,,${ }^{1,5}$ K. Göbel, ${ }^{1}$ G. Hampel, ${ }^{4}$ M. Heftrich, ${ }^{1}$ F. Käppeler, ${ }^{6}$ C. Lederer, ${ }^{7}$ M. Mikorski, ${ }^{1}$ R. Plag, ${ }^{1}$ R. Reifarth, ${ }^{1}$ C. Stieghorst,${ }^{4}$ S. Schmidt,,${ }^{1}$ D. Schumann, ${ }^{3}$ Z. Slavkovská, ${ }^{1}$ K. Sonnabend, ${ }^{1}$ A. Wallner, ${ }^{8}$ M. Weigand, ${ }^{1}$ N. Wiehl, ${ }^{4}$ and S. Zauner ${ }^{4}$

${ }^{1}$ Goethe Universität Frankfurt, Frankfurt, Germany

${ }^{2}$ Technische Universität Wien, Vienna, Austria

${ }^{3}$ Paul Scherrer Institut, Villigen, Switzerland

${ }^{4}$ Johannes Gutenberg-Universität Mainz, Mainz, Germany

${ }^{5}$ GSI Helmholtzzentrum für Schwerionenforschung, Darmstadt, Germany

${ }^{6}$ Karlsruhe Institute of Technology, Karlsruhe, Germany

${ }^{7}$ University of Edinburgh, Edinburgh, United Kingdom

${ }^{8}$ Australian National University, Canberra, Australia

(Received 26 January 2015; published 23 July 2015)

\begin{abstract}
Background: Fifty percent of the heavy element abundances are produced via slow neutron capture reactions in different stellar scenarios. The underlying nucleosynthesis models need the input of neutron capture cross sections.

Purpose: One of the fundamental signatures for active nucleosynthesis in our galaxy is the observation of long-lived radioactive isotopes, such as ${ }^{60} \mathrm{Fe}$ with a half-life of $2.60 \times 10^{6} \mathrm{yr}$. To reproduce this $\gamma$ activity in the universe, the nucleosynthesis of ${ }^{60} \mathrm{Fe}$ has to be understood reliably.

Methods: An ${ }^{60} \mathrm{Fe}$ sample produced at the Paul Scherrer Institut (Villigen, Switzerland) was activated with thermal and epithermal neutrons at the research reactor at the Johannes Gutenberg-Universität Mainz (Mainz, Germany).

Results: The thermal neutron capture cross section has been measured for the first time to $\sigma_{\text {th }}=0.226\left({ }_{-0.049}^{+0.044}\right) \mathrm{b}$. An upper limit of $\sigma_{\mathrm{RI}}<0.50 \mathrm{~b}$ could be determined for the resonance integral.

Conclusions: An extrapolation towards the astrophysically interesting energy regime between $k T=10$ and 100 $\mathrm{keV}$ illustrates that the $s$-wave part of the direct capture component can be neglected.
\end{abstract}

DOI: 10.1103/PhysRevC.92.015806

PACS number(s): 25.40.Lw, 26.20.Kn, 27.50.+e, 28.20.Ka

\section{INTRODUCTION}

The decays of the unstable isotopes ${ }^{60} \mathrm{Fe}\left(t_{1 / 2}=2.60\right.$ Myr [1]) and ${ }^{26} \mathrm{Al}\left(t_{1 / 2}=0.717 \mathrm{Myr}\right.$ [2]) in the Milky Way, which have been observed with satellite-based $\gamma$-ray telescopes $[3,4]$, are considered as a clear signature of ongoing stellar nucleosynthesis [5].

The production of ${ }^{60} \mathrm{Fe}$ in the slow neutron capture process ( $s$-process) [5] is hampered by the rather short-lived precursor ${ }^{59} \mathrm{Fe}\left(t_{1 / 2}=44.495 \mathrm{~d}[6]\right)$, which acts as a branch point of the $s$-process path as illustrated in Fig. 1. Accordingly, high neutron densities are required to avoid that the reaction flow bypasses ${ }^{60} \mathrm{Fe}$ via the decay of ${ }^{59} \mathrm{Fe}$. Once ${ }^{60} \mathrm{Fe}$ is reached, it can also be destroyed by neutron capture or-on longer time scales-by $\beta^{-}$decay. High neutron densities are generally accompanied by very high temperatures, but the synthesis of ${ }^{60} \mathrm{Fe}$ requires an upper limit of about $2 \times 10^{9} \mathrm{~K}\left(T_{9}=2\right)$, because photodisintegration reactions such as ${ }^{60} \mathrm{Fe}(\gamma, n)$ and ${ }^{59} \mathrm{Fe}(\gamma, n)$ start to dominate otherwise.

There are two different astrophysical scenarios where ${ }^{60} \mathrm{Fe}$ can be produced [7]: during the He-shell burning phase in low-mass thermally pulsing asymptotic giant branch (AGB) stars and during the convective $\mathrm{C}$-shell burning in massive presupernova stars. In AGB stars, neutron densities of $10^{10} \mathrm{~cm}^{-3}$

*heftrich@iap.uni-frankfurt.de and temperatures around $2.5 \times 10^{8} \mathrm{~K}\left(T_{8}=2.5\right)$ are reached, whereas in massive stars neutron densities of $10^{12} \mathrm{~cm}^{-3}$ at temperatures of up to $T_{8}=10$ during C-shell burning are reached [8]. According to detailed stellar model calculations by Limongi and Chieffi [7], about $65 \%$ of the total yield of ${ }^{60} \mathrm{Fe}$ is in fact synthesized in the presupernova stage of massive stars and $18 \%$ is contributed by the He burning shell of less massive stars. A third major component is eventually produced by explosive shell burning during the supernova itself. These contributions to the total ${ }^{60} \mathrm{Fe}$ yield are strongly affected by the respective masses and metallicities of the stars involved and may vary correspondingly.

A crucial input for the production of ${ }^{60} \mathrm{Fe}$ in AGB stars and massive presupernova stars are the neutron capture cross sections at the respective stellar temperatures. So far, an activation measurement of the ${ }^{60} \mathrm{Fe}(n, \gamma){ }^{61} \mathrm{Fe}$ cross section at neutron energies corresponding to a thermal energy of $k T=25 \mathrm{keV}$ (typical for AGB stars) was performed at Forschungszentrum Karlsruhe, Germany. The Maxwellian averaged cross section (MACS) at $k T=30 \mathrm{keV}$ was determined to $5.15 \pm 1.4 \mathrm{mb}$ [9]. The direct capture (DC) component of the cross section at this temperature constitutes important information for the extrapolation towards the astrophysically interesting temperatures in massive stars around $k T=90 \mathrm{keV}$. In this respect, the thermal cross section provides a constraint for the $s$-wave component of the DC cross section. Therefore, the previously unknown thermal cross section of ${ }^{60} \mathrm{Fe}$ was 


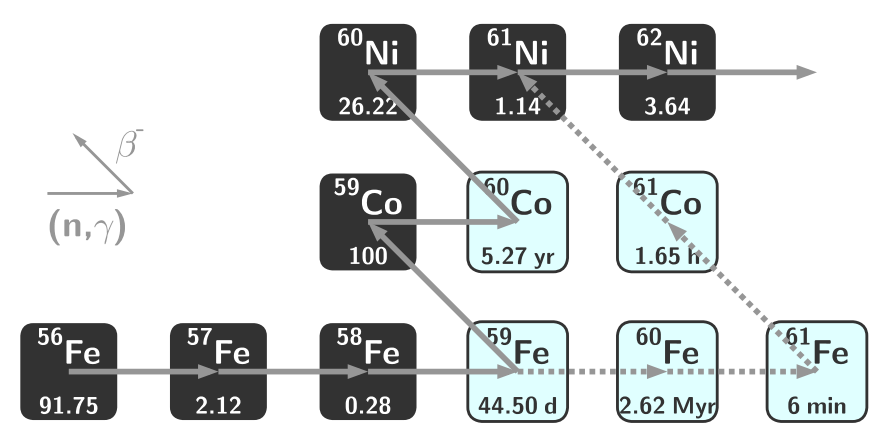

FIG. 1. (Color online) The $s$-process reaction path between Fe and $\mathrm{Ni}$. The isotope ${ }^{60} \mathrm{Fe}$ is produced via a sequence of $(n, \gamma)$ reactions starting at the stable iron isotopes. Because of the short half-life of ${ }^{59} \mathrm{Fe}\left(t_{1 / 2} \approx 45 \mathrm{~d}\right)$, the production of ${ }^{60} \mathrm{Fe}$ depends critically on the stellar neutron density.

measured using the irradiation facility at the TRIGA (Training, Research, Isotopes, General Atomic) type research reactor at Johannes Gutenberg-Universität Mainz (Mainz, Germany) [10,11].

\section{EXPERIMENT}

The ${ }^{60} \mathrm{Fe}$ sample was produced at the Paul Scherrer Insitut (PSI) in Villigen, Switzerland [12]. To compensate for the limited amount of ${ }^{60} \mathrm{Fe}$ the only possible method for the determination of the neutron capture cross section was an integral activation measurement at high neutron fluxes. Compared to the more generally applicable time-offlight technique, the activation method has the advantage of excellent sensitivity [8], which allows neutron capture measurements even on very small samples [13,14]. This technique has the additional advantage that it does not require isotopically enriched samples, because the capture reactions can be identified via the $\gamma$-decay characteristics of the product nucleus ${ }^{61} \mathrm{Fe}$.

\section{A. Measurements and calibration}

The induced activities were measured using a high-purity germanium (HPGe) detector (CANBERRA-GX7020) with a relative efficiency of $72.3 \%$. The output signals from the preamplifier were converted with a flash-ADC (CAEN Module V1724). The dead-time corrections were determined using a ${ }^{137} \mathrm{Cs}$ sample, which was placed at a fixed distant position during all activity measurements. The corresponding corrections were negligibly small. Because of the contamination of ${ }^{55} \mathrm{Fe}$ in the ${ }^{60} \mathrm{Fe}$ sample, the activity of ${ }^{55} \mathrm{Fe}$ was suppressed by a lead foil $1 \mathrm{~mm}$ in thickness.

The efficiency was determined by a calibrated solution containing the standard single- or double-line $\gamma$-ray emitters ${ }^{60} \mathrm{Co},{ }^{85} \mathrm{Sr},{ }^{88} \mathrm{Y},{ }^{113} \mathrm{Sn},{ }^{137} \mathrm{Cs},{ }^{139} \mathrm{Ce}$, and ${ }^{203} \mathrm{Hg}$. The uncertainty of the $\gamma$-emission rate was given with $3 \%(2 \sigma)$. This multinuclide solution was absorbed in a pure graphite disk $6 \mathrm{~mm}$ in diameter and $1 \mathrm{~mm}$ in thickness to match the properties of the ${ }^{60} \mathrm{Fe}$ sample used in the measurement (see below). For all $\gamma$-activity measurements, the samples were placed $7.4 \mathrm{~mm}$ in front of the Ge crystal. Because of the small distance between the sample and the detector, cascade corrections were necessary for the decays of ${ }^{60} \mathrm{Co}$ and ${ }^{89} \mathrm{Y}$. Those corrections were based on the simulations performed using the GEANT3.21 package $[15,16]$. The corresponding correction for the emission line of ${ }^{60} \mathrm{Co}$ at an energy of $1173 \mathrm{keV}$ was $30 \%$ and at $1332 \mathrm{keV}$ it was $31 \%$. For ${ }^{88} \mathrm{Y}$ at energies of 898 and $1836 \mathrm{keV}$, the corresponding corrections were $27 \%$ and $29 \%$, respectively. As shown in Fig. 2 the measured efficiencies could be reproduced within the experimental uncertainties of $\pm 3.5 \%$ over the energy range from 150 to $1900 \mathrm{keV}$ by the expression

$$
\epsilon_{\gamma}=A \exp \left\{-B \ln \left[E_{\gamma}-C+D \exp \left(F \times E_{\gamma}\right)\right]\right\} .
$$

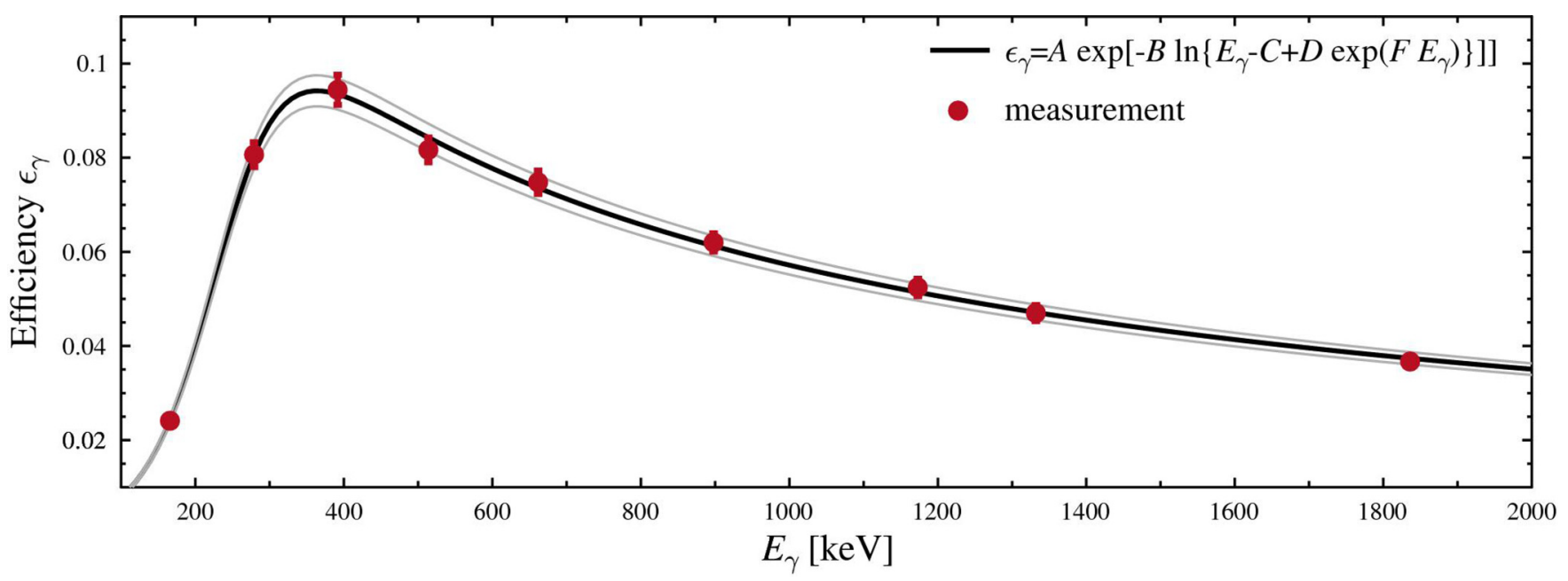

FIG. 2. (Color online) The detection efficiency of the HPGe detector used for the activity measurements in Mainz. The efficiency used for determination of the number of ${ }^{60} \mathrm{Fe}$ particles is given in Table I. The solid line shows the least-squares fit to interpolate between the data points of the calibrated solution (red circles). For ${ }^{88} \mathrm{Y}$ and ${ }^{60} \mathrm{Co}$ the data points were corrected for cascade effects using the GEANT-3.21 package [15,16]. The grey band represents the uncertainty of the fit. 


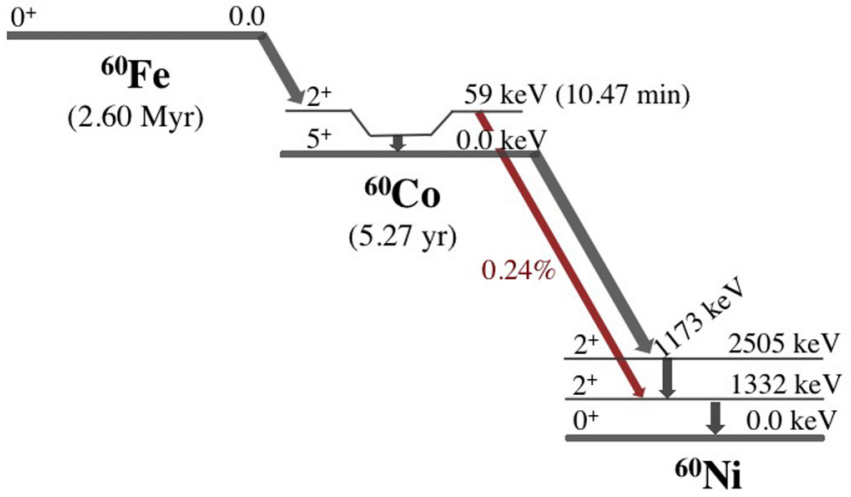

FIG. 3. (Color online) The activity of the ${ }^{60} \mathrm{Fe}$ sample was determined by the $\gamma$-ray cascade with energies of 1173 and $1332 \mathrm{keV}$ emitted after the $\beta$ decay of the daughter nucleus ${ }^{60} \mathrm{Co}$. Data are from Refs. [17,21].

\section{B. Sample preparation}

The ${ }^{60} \mathrm{Fe}$ was extracted from slices of a cylindrical copper beam dump, which was previously irradiated with $590-\mathrm{MeV}$ protons at the PSI [12]. In addition to ${ }^{60} \mathrm{Fe}$ activity, the initial copper sample of $3 \mathrm{~g}$ also contained $150 \mathrm{MBq}$ of ${ }^{60} \mathrm{Co}, 100$ $\mathrm{MBq}$ of ${ }^{55} \mathrm{Fe}$, and $2 \mathrm{MBq}$ of ${ }^{44} \mathrm{Ti}$. Details of the chemical separation of the ${ }^{60} \mathrm{Fe}$ fraction are described in Ref. [12]. The final purification was performed shortly before the experiment using liquid-liquid extraction into methyl isobutyl ketone from $7 \mathrm{M} \mathrm{HCl}$ solution and following back-extraction with diluted $\mathrm{HCl}$. This solution was evaporated on a graphite disk $6 \mathrm{~mm}$ in diameter and $1 \mathrm{~mm}$ in thickness.

The number of ${ }^{60} \mathrm{Fe}$ atoms in the sample was determined via the increasing ${ }^{60} \mathrm{Co}$ activity $\left(t_{1 / 2}=5.272 \mathrm{yr}[17]\right)$ according to Fig. 3. The activity of ${ }^{60} \mathrm{Co}$ nuclei increases as

$$
A\left({ }^{60} \mathrm{Co}\right)=\left\{1-\exp \left[-\lambda\left({ }^{60} \mathrm{Co}\right) \mathrm{t}\right]\right\} \mathrm{A}\left({ }^{60} \mathrm{Fe}\right),
$$

where $\lambda$ is the decay constant. The related $\gamma$ activity at time $t$ can be derived using the integrated line strength $C_{\gamma}$ :

$$
A_{t}\left({ }^{60} \mathrm{Co}\right)=\frac{C_{\gamma}}{0.9976 \epsilon_{\gamma} I_{\gamma}} \frac{\lambda}{\exp \{-\lambda t\}-\exp \left\{-\lambda\left(t+t_{\mathrm{m}}\right)\right\}},
$$

where $\lambda$ is the decay constant of ${ }^{60} \mathrm{Co}$ and with the factor $0.9976 \pm 0.0003$ [17] for the fraction of ${ }^{60} \mathrm{Co}^{m}$, which decays to the ground state of ${ }^{60} \mathrm{Co}$, the measurement time $t_{m}$, and the detection efficiencies for the 1173- and 1332-keV $\gamma$ transitions, respectively. For the analysis of the emission line at an energy of $1332 \mathrm{keV}$, the decay of ${ }^{60} \mathrm{Co}^{m}$ has to be corrected. The decay intensities $I_{\gamma}$ and efficiencies $\epsilon_{\gamma}$ are listed in Table I, which summarizes all the decay characteristics adopted in the data analysis. With $A=\lambda N$, the number of ${ }^{60} \mathrm{Fe}$ atoms becomes

$$
N\left({ }^{60} \mathrm{Fe}\right)=\frac{\mathrm{A}_{\mathrm{t}}\left({ }^{60} \mathrm{Co}\right)}{1-\exp \left\{-\lambda\left({ }^{60} \mathrm{Co}\right) \mathrm{t}\right\}} \frac{1}{\lambda\left({ }^{60} \mathrm{Fe}\right)} .
$$

The activity measurement of ${ }^{60} \mathrm{Co}$ was carried out at the Goethe-Universität Frankfurt 38 months after the purification using an HPGe detector of $98 \%$ relative efficiency (see Fig. 4). Background due to the activity of the ${ }^{55} \mathrm{Fe}$ contamination in the sample was suppressed by a lead foil $1 \mathrm{~mm}$ in thickness. The number of ${ }^{60} \mathrm{Fe}$ atoms in the sample,

$$
N\left({ }^{60} \mathrm{Fe}\right)=\left(7.77 \pm 0.11_{\text {stat }} \pm 0.42_{\text {syst }}\right) \times 10^{14},
$$

was determined as a weighted average comprising both ${ }^{60} \mathrm{Co}$ lines. The systematic uncertainty was determined by the $\gamma$-ray detection efficiency, the decay intensities, and the half-life (see Table I). As the half-life of ${ }^{60} \mathrm{Fe}$, the value $t_{1 / 2}=2.60 \pm 0.05$ Myr [1] was used.

\section{Reactor activations}

In view of the short half-life of the produced ${ }^{61} \mathrm{Fe}$ nuclei $\left(t_{1 / 2}=5.98 \mathrm{~min}\right.$ [20]), the activations at the TRIGA research reactor were performed using a pneumatic transport system between the irradiation position and the counting room $[10,11]$.

The ${ }^{60} \mathrm{Fe}$ sample was activated for $t_{a}=10 \mathrm{~min}$ with and without cadmium foils surrounding the sample in both cases. This so-called cadmium-difference-method allows the distinction between the thermal neutron capture cross section and the resonance integral, which takes into account the epithermal component of the reactor neutron spectrum. The reactor spectrum can be described as the sum of a thermal com-

\begin{tabular}{|c|c|c|c|c|c|}
\hline Isotope & $t_{1 / 2}$ & $E_{\gamma}(\mathrm{keV})$ & $I_{\gamma}(\%)$ & Reference & $\epsilon_{\gamma}$ \\
\hline${ }^{60} \mathrm{Co}$ & $1925.28 \pm 0.14 \mathrm{~d}$ & $1332.492 \pm 0.004$ & $99.9826 \pm 0.0006$ & [17] & $0.022 \pm 0.0002^{\mathrm{a}}$ \\
\hline \multirow[t]{2}{*}{${ }^{95} \mathrm{Zr}$} & \multirow[t]{2}{*}{$64.032 \pm 0.006 \mathrm{~d}$} & $724.195 \pm 0.004$ & $44.27 \pm 0.22$ & \multirow[t]{2}{*}{ [19] } & $0.070 \pm 0.001^{\mathrm{b}}$ \\
\hline & & $756.728 \pm 0.012$ & $54.38 \pm 0.22$ & & $0.068 \pm 0.002^{b}$ \\
\hline${ }^{61} \mathrm{Fe}$ & $5.98 \pm 0.06 \mathrm{~min}$ & $297.90 \pm 0.07$ & $22.24 \pm 2.88$ & {$[20]$} & $0.087 \pm 0.002^{\mathrm{b}}$ \\
\hline
\end{tabular}
ponent, i.e., a Maxwell-Boltzmann distribution corresponding to $k T=25.3 \mathrm{meV}$, and an epithermal component following

TABLE I. Decay characteristics and efficiency of $\gamma$ emission of the investigated nuclei.

${ }^{\text {a }}$ Measured with a HPGe detector at Goethe-Universität Frankfurt (used for ${ }^{60} \mathrm{Fe}$ determination).

${ }^{\mathrm{b}}$ Measured with a different HPGe detector at the research reactor at Johannes Gutenberg-Universität Mainz (used for ${ }^{61} \mathrm{Fe}$ and zirconium determination). 


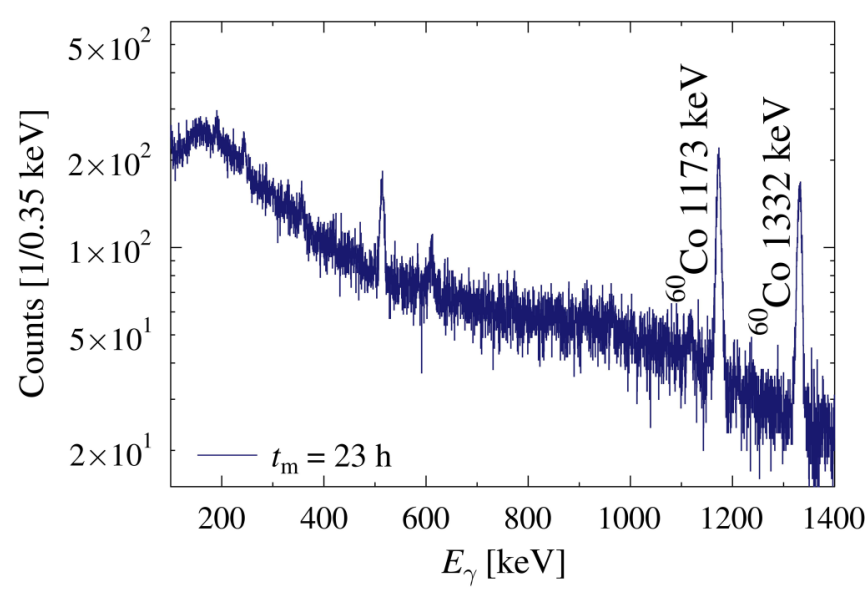

FIG. 4. (Color online) $\gamma$-ray spectrum for the determination of the ${ }^{60} \mathrm{Co}$ activity taken 34 months after purification of the sample. The measurement time was $t_{m}=23 \mathrm{~h}$.

a $1 / E$ dependence. Due to the very large thermal capture cross section of cadmium, a proper cadmium shielding of the sample results in significantly different responses to thermal and epithermal neutrons. In the ideal case, all thermal neutrons would be absorbed in the cadmium, while the epithermal spectrum would remain undisturbed.

The number of product nuclei after the activation $N\left({ }^{A+1} X\right)$ can be expressed in terms of the thermal cross section $\sigma_{\text {th }}$, the resonance integral $\sigma_{\mathrm{RI}}\left(\sigma_{\mathrm{RI}}=\int_{E_{\text {cutoff }}}^{2 \mathrm{MeV}} \sigma(E) / E \mathrm{~d} E\right.$ with the cutoff energy $\left.E_{\text {cutoff }} \approx 90 \mathrm{meV}\right)$, and the epithermal $\left(\Phi_{\text {epi }}\right)$ and thermal neutron fluences $\left(\Phi_{\mathrm{th}}\right)$ in units of $\mathrm{cm}^{-2}$,

$$
N\left({ }^{A+1} X\right)=N\left({ }^{A} X\right)\left(\Phi_{\mathrm{th}} \sigma_{\mathrm{th}}+\Phi_{\mathrm{epi}} \sigma_{\mathrm{RI}}\right),
$$

where $N\left({ }^{A} X\right)$ is the number of target nuclei in the irradiated sample. Natural zirconium provides a well-suited monitor for the epithermal and the thermal flux. The activation of the isotopes ${ }^{94} \mathrm{Zr}$ and ${ }^{96} \mathrm{Zr}$ results in significantly different ratios $\sigma_{\mathrm{RI}} / \sigma_{\mathrm{th}}$ (Table II). The uncertainties in the number of $\mathrm{Zr}$ atoms are due to the sample weight $(0.2 \%)$ and to the isotopic abundances $\left(1.6 \%\right.$ and $3.2 \%$ for ${ }^{94} \mathrm{Zr}$ and ${ }^{96} \mathrm{Zr}$, respectively) [23]. Two sets of $\mathrm{Zr}$ foils were used in the activations with and without cadmium shielding.

\section{RESULTS}

\section{A. Determination of the neutron fluence}

The zirconium foils used as neutron monitors were $0.127 \mathrm{~mm}$ in thickness and $6 \mathrm{~mm}$ in diameter. The foils

TABLE II. Number of atoms $N$ (in units of $10^{19}$ ) and cross sections (in barns) of the neutron fluence monitors ${ }^{94} \mathrm{Zr}$ and ${ }^{96} \mathrm{Zr}$. Cross sections were obtained from Ref. [22].

\begin{tabular}{lcc}
\hline \hline & \multicolumn{1}{c}{${ }^{94} \mathrm{Zr}$} & ${ }^{96} \mathrm{Zr}$ \\
\hline$N$ & $2.7331 \pm 0.044$ & $0.440 \pm 0.014$ \\
$N_{\mathrm{Cd}}$ & $2.7101 \pm 0.044$ & $0.437 \pm 0.014$ \\
$\sigma_{\text {th }}$ & $0.0494 \pm 0.0017$ & $0.0229 \pm 0.0010$ \\
$\sigma_{\mathrm{RI}}$ & $0.280 \pm 0.010$ & $5.28 \pm 0.11$ \\
\hline \hline
\end{tabular}

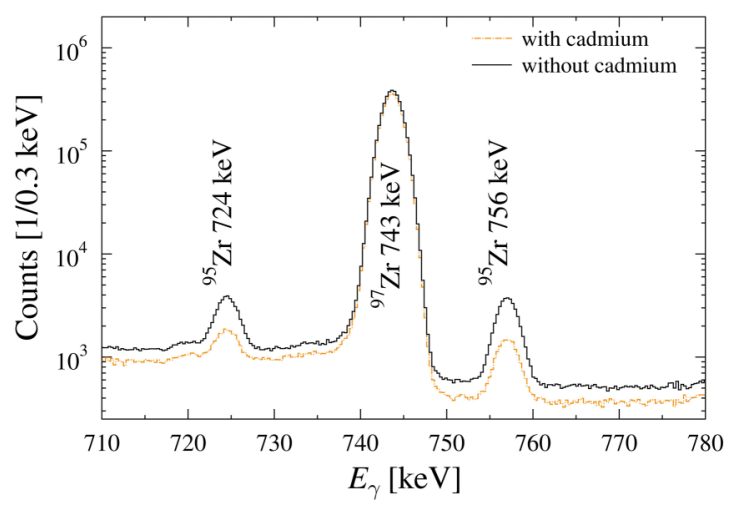

FIG. 5. (Color online) The $\gamma$-ray spectra of the $\mathrm{Zr}$ monitor foils used for the neutron fluence analysis normalized for different measuring times. Due to the smaller capture cross section of ${ }^{96} \mathrm{Zr}$ in the thermal energy regime, the cadmium shielding affects the ${ }^{97} \mathrm{Zr}$ signature to a much lower degree than that of ${ }^{95} \mathrm{Zr}$.

were thin enough that neutron self-absorption losses during $\gamma$ spectroscopy could be neglected. The fluences in units of $1 / \mathrm{cm}^{2}$ are

$$
\Phi_{\mathrm{epi}}=\frac{N^{97}-N^{96} \sigma_{\mathrm{th}}^{96} \Phi_{\mathrm{th}}}{N^{96} \sigma_{\mathrm{RI}}^{96}}
$$

and

$$
\Phi_{\text {th }}=\frac{N^{96} N^{95} \sigma_{\mathrm{RI}}^{96}-N^{94} N^{97} \sigma_{\mathrm{RI}}^{94}}{N^{94} N^{96}\left[\sigma_{\mathrm{RI}}^{96} \sigma_{\text {th }}^{94}-\sigma_{\mathrm{RI}}^{94} \sigma_{\mathrm{th}}^{96}\right]},
$$

where the indices are referring to the various $\mathrm{Zr}$ isotopes. Figure 5 shows the $\gamma$-ray spectra of the monitor foils normalized to equal neutron fluence. Because of the small neutron capture cross section of ${ }^{96} \mathrm{Zr}$ in the thermal energy regime, the ${ }^{97} \mathrm{Zr}$ signal is only marginally affected by the cadmium shielding, whereas ${ }^{95} \mathrm{Zr}$ exhibits a clear effect due to the larger thermal cross section of ${ }^{94} \mathrm{Zr}$. The number of produced $\mathrm{Zr}$ nuclei is

$$
N\left({ }^{i} X\right)=\frac{C_{\gamma}}{\epsilon_{\gamma} I_{\gamma} f_{a} f_{w} f_{m}},
$$

where

$$
\begin{gathered}
f_{a}=\frac{1-\exp \left(-\lambda_{i} t_{a}\right)}{\lambda_{i} t_{a}}, \\
f_{w}=\exp \left(-\lambda_{i} t_{\mathrm{w}}\right), \\
f_{m}=1-\exp \left(-\lambda_{i} t_{\mathrm{m}}\right)
\end{gathered}
$$

are the corrections for the decay during the activation $\left(f_{a}\right)$, during the waiting time between activation and measurement $\left(f_{w}\right)$, and during the measurement $\left(f_{m}\right)$, respectively. The correction for the dead time was of the order of $0.5 \%$. The systematic uncertainty was again determined by the $\gamma$ efficiencies, the decay intensities, and the half-lives (Table I). The resulting neutron fluences for the two activations are listed in Table III. 
TABLE III. The number of $\mathrm{Zr}$ nuclei produced in the activations and the resulting neutron fluences without and with cadmium shielding. ${ }^{\text {a }}$

\begin{tabular}{lcc}
\hline \hline & Without Cd & With Cd \\
\hline$N\left({ }^{95} \mathrm{Zr}\right)^{\mathrm{b}}$ & $1.517 \pm 0.005 \pm 0.018$ & $0.510 \pm 0.006 \pm 0.008$ \\
$N\left({ }^{97} \mathrm{Zr}\right)^{\mathrm{b}}$ & $1.171 \pm 0.001 \pm 0.018$ & $1.067 \pm 0.001 \pm 0.016$ \\
$\Phi_{\text {th }}{ }^{\mathrm{c}}$ & $8.60 \pm 0.03 \pm 0.38$ & $1.21 \pm 0.01 \pm 0.16$ \\
$\Phi_{\text {epi }}{ }^{\mathrm{c}}$ & $0.467 \pm 0.002 \pm 0.014$ & $0.458 \pm 0.005 \pm 0.014$ \\
\hline \hline
\end{tabular}

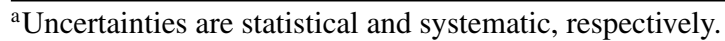

${ }^{\mathrm{b}}$ In units of $10^{9}$.

${ }^{\mathrm{c}}$ In units of $10^{14} \mathrm{~cm}^{-2}$.

\section{B. Thermal $(n, \gamma)$ cross section of ${ }^{60} \mathrm{Fe}$}

The $\gamma$ spectrum measured after the activation of the ${ }^{60} \mathrm{Fe}$ sample without cadmium shielding (Fig. 6) clearly exhibits the $\gamma$ transitions of ${ }^{61} \mathrm{Fe}$ at 297.9, 1027, and $1205 \mathrm{keV}$. However, only the last two were used in the analysis because of the poor signal-to-background ratio of the $298-\mathrm{keV}$ line. The systematic uncertainty is calculated by the error of the efficiency, the $I_{\gamma}$, the half-lives, and the neutron fluences (see Tables I, III, and IV). In the corresponding spectrum measured after the activation with cadmium shielding, the ${ }^{61} \mathrm{Fe}$ lines are completely missing as illustrated in Fig. 7 for the 1027-keV line as an example. In this case, only an upper limit can
TABLE IV. The number of ${ }^{61} \mathrm{Fe}$ nuclei (in units of $10^{5}$ ) produced in the activations.

\begin{tabular}{|c|c|c|}
\hline \multirow{2}{*}{$\begin{array}{l}\gamma \text {-ray energy } \\
(\mathrm{keV})\end{array}$} & \multicolumn{2}{|c|}{$N\left({ }^{61} \mathrm{Fe}\right)^{\mathrm{a}}$} \\
\hline & Without Cd & With Cd \\
\hline 1027 & $1.54 \pm 0.19 \pm 0.18$ & $<0.179$ \\
\hline 1205 & $1.48 \pm 0.20 \pm 0.16$ & $<0.206$ \\
\hline Weighted average & $1.51 \pm 0.14 \pm 0.24$ & $<0.179^{\mathrm{b}}$ \\
\hline
\end{tabular}

${ }^{a}$ Uncertainties are statistical and systematic, respectively.

${ }^{\mathrm{b}}$ Adopted upper limit for further discussion.

be determined for the resonance integral. The numbers of produced ${ }^{61} \mathrm{Fe}$ nuclei are listed in Table IV.

The number ratio of ${ }^{61} \mathrm{Fe}$ and ${ }^{60} \mathrm{Fe}$ after the activation without cadmium is

$$
N\left({ }^{61} \mathrm{Fe}\right) / N\left({ }^{60} \mathrm{Fe}\right)=\Phi_{\text {th }} \sigma_{\text {th }}+\Phi_{\text {epi }} \sigma_{\mathrm{RI}} .
$$

The thermal cross section

$$
\sigma_{\mathrm{th}}\left({ }^{60} \mathrm{Fe}\right)=\frac{N\left({ }^{61} \mathrm{Fe}\right)}{N\left({ }^{60} \mathrm{Fe}\right)} \frac{1}{\Phi_{\mathrm{th}}}-\sigma_{\mathrm{RI}} \frac{\Phi_{\mathrm{epi}}}{\Phi_{\mathrm{th}}}
$$

is determined by the number of sample atoms $N\left({ }^{60} \mathrm{Fe}\right)$ (Sec. II B), the neutron fluences $\Phi_{\text {th }}$ and $\Phi_{\text {epi }}$ from the $\mathrm{Zr}$ monitor measurements (Table III), and the number of ${ }^{61} \mathrm{Fe}$ nuclei produced during the activations $N\left({ }^{61} \mathrm{Fe}\right)$ (Table IV).
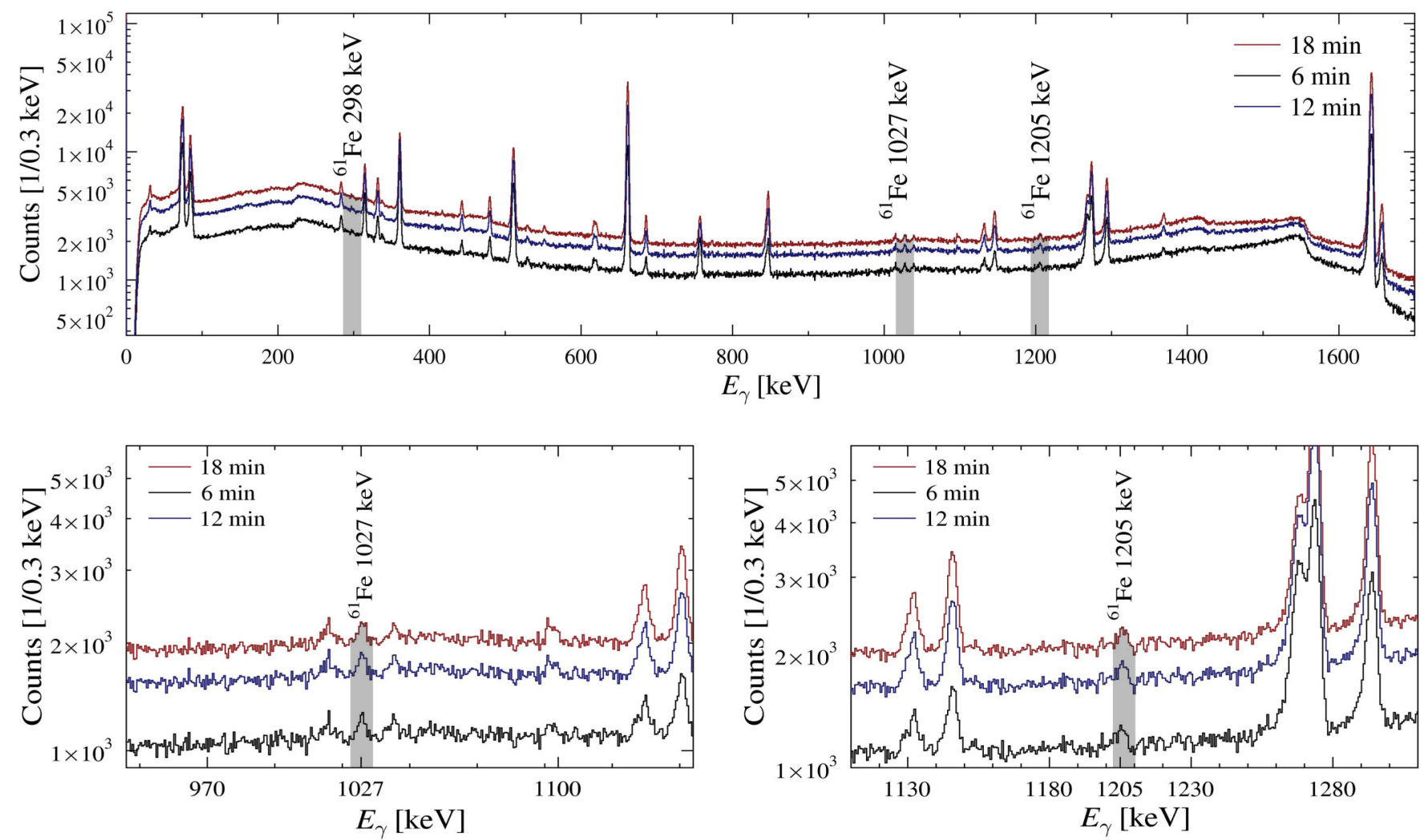

FIG. 6. (Color online) The $\gamma$-ray spectrum of the activation without cadmium shielding measured for one, two, and three half-lives of ${ }^{61} \mathrm{Fe}$, respectively. The upper panel provides an overview, and the lower panels show a zoom into the regions of the ${ }^{61} \mathrm{Fe}$ lines at 1027 and $1205 \mathrm{keV}$. The background stems from the activation of the contaminants of the ${ }^{60} \mathrm{Fe}$ sample. 


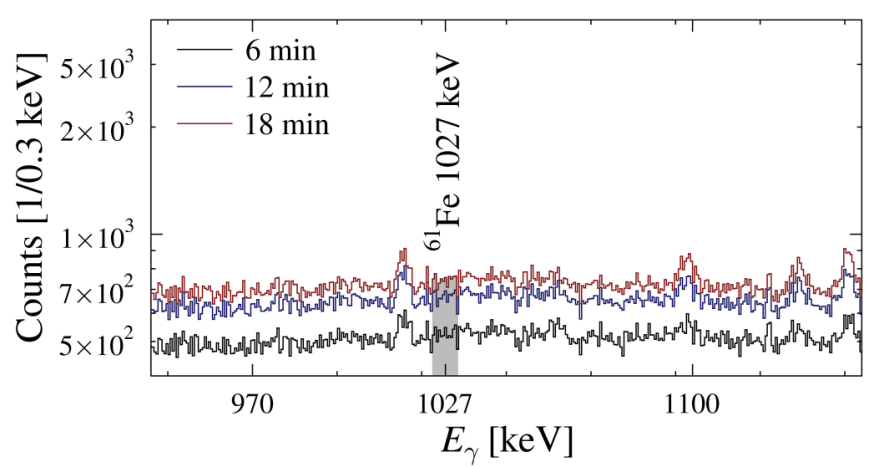

FIG. 7. (Color online) A detailed view into the region around $1027 \mathrm{keV}$ of the $\gamma$-ray spectrum after activation with the cadmium shielding, illustrating the absence of ${ }^{61} \mathrm{Fe}$ lines. Therefore, only an upper limit can be deduced from the data.

The resonance integral

$$
\sigma_{\mathrm{RI}}=\frac{\frac{\left.N^{\mathrm{Cd}(61} \mathrm{Fe}\right)}{N\left({ }^{60} \mathrm{Fe}\right)}-\frac{\Phi_{\mathrm{th}}^{\mathrm{Cd}}}{\Phi_{\mathrm{th}}} \frac{N\left({ }^{61} \mathrm{Fe}\right)}{N\left({ }^{60} \mathrm{Fe}\right)}}{\Phi_{\mathrm{epi}}^{\mathrm{Cd}}-\frac{\Phi_{\mathrm{cd}}^{\mathrm{Cd}} \Phi_{\mathrm{epi}}}{\Phi_{\mathrm{th}}}},
$$

is obtained accordingly. Because the epithermal fluences were almost equal in both activations, and because the number of ${ }^{61} \mathrm{Fe}$ nuclei produced with the cadmium absorber is much smaller than that produced without absorber, an upper limit for the resonance integral can be defined as

$$
\sigma_{\mathrm{RI}}<\frac{N^{\mathrm{Cd}}\left({ }^{61} \mathrm{Fe}\right)}{N\left({ }^{60} \mathrm{Fe}\right)} \frac{1}{\Phi_{\mathrm{epi}}^{\mathrm{Cd}}} .
$$

Assuming a $1 \sigma$ confidence level as a constraint for the resonance integral derived from the $1027-\mathrm{keV}$ line, one finds

$$
0<\sigma_{\mathrm{RI}}<0.50 \mathrm{~b}
$$

for calculating the thermal cross section using Eq. (13).
A variation of the resonance integral within these limits affects the thermal cross section by about $10 \%$. We assume the resonance integral here explicitly as

$$
\sigma_{\mathrm{RI}}=0.00_{-0.00}^{+0.50} \mathrm{~b},
$$

consistent with Eq. (16), and treat this range as a systematic uncertainty. Should the resonance integral be improved in the future, the thermal cross section could be reevaluated accordingly. With Eqs. (13) and (17) the thermal cross section of ${ }^{60} \mathrm{Fe}$ becomes

$$
\sigma_{\text {th }}\left({ }^{60} \mathrm{Fe}\right)=\left[0.226 \pm 0.021_{\text {stat }}\left(\begin{array}{l}
+0.039 \\
-0.045
\end{array}\right)_{\text {syst }}\right] \text { b. }
$$

\section{SUMMARY AND DISCUSSION}

Within this work, we characterized the ${ }^{60} \mathrm{Fe}$ sample to contain $N\left({ }^{60} \mathrm{Fe}\right)=\left(7.77 \pm 0.11_{\text {stat }} \pm 0.42_{\text {syst }}\right) \times 10^{14}$ atoms. Using the cadmium-difference-method two activations of that sample have been performed at the TRIGA research reactor at Johannes Gutenberg-Universität Mainz. The neutron capture cross section of ${ }^{60} \mathrm{Fe}$ at thermal energies and an experimental upper limit for the resonance integral were determined for the first time:

$$
\sigma_{\text {th }}\left({ }^{60} \mathrm{Fe}\right)=0.226\left(\begin{array}{c}
+0.044 \\
-0.049
\end{array}\right) \mathrm{b}
$$

and

$$
\sigma_{\mathrm{RI}}<0.50 \mathrm{~b} .
$$

Figure 8 shows a comparison of our data with evaluated cross sections (TENDL-2014 [24]) and the only experimental value of $5.7 \pm 1.4 \mathrm{mb}$ at $k T=25 \mathrm{keV}$ so far $[9,25]$. Under the assumption that the MACS in the meV regime is dominated by an $s$-wave DC component, an extrapolation towards higher energies via $1 / \sqrt{E}$ is possible. Together with the measurement

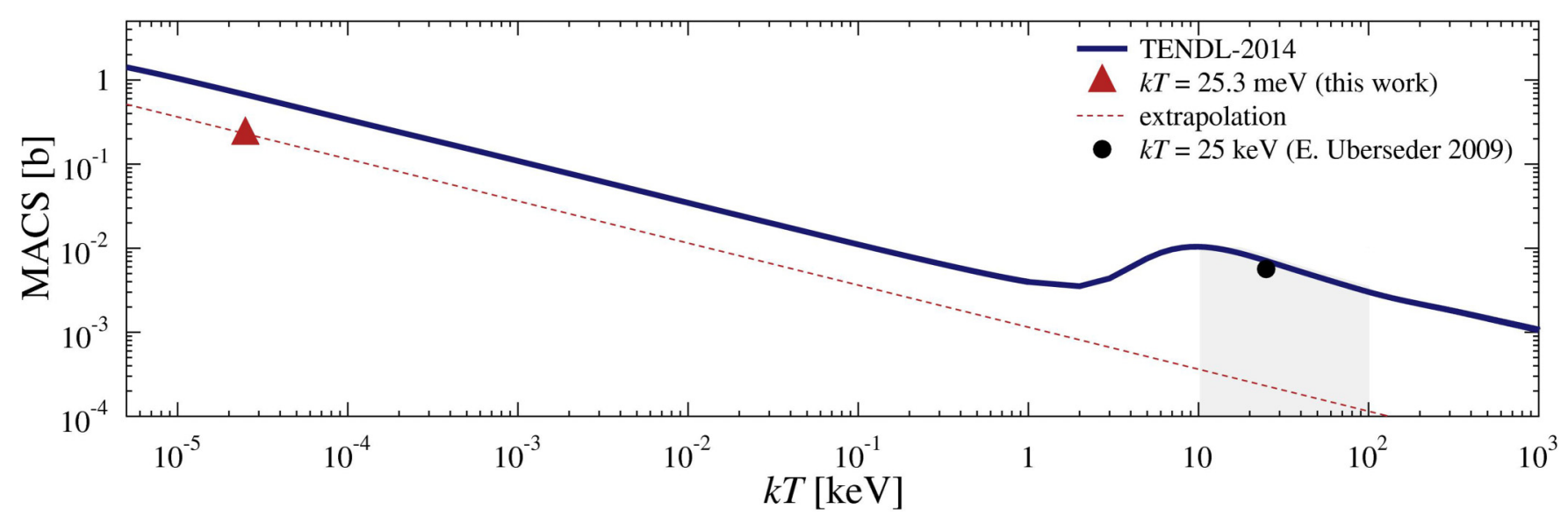

FIG. 8. (Color online) The Maxwellian averaged cross section for ${ }^{60} \mathrm{Fe}(n, \gamma)$. The present measurement of the cross section at $k T=$ $25.3 \mathrm{meV}$ (red triangle) and an $0.00115 / \sqrt{E}$ extrapolation to the astrophysical energy regime are indicated by the solid red triangle and the dashed red line, respectively. This extrapolation can be used to estimate the DC of the MACS at $k T=25 \mathrm{keV}$ (black dot) [9]. The astrophysical energy regime from $k T=10 \mathrm{keV}-100 \mathrm{keV}$ (grey box) is clearly dominated by the resonant capture contribution. Below about $1 \mathrm{keV}$, the MACS based on the most recent version of the TENDL library (TENDL-2014 [24], blue line) are a factor of 3 above the current measurement. This indicates that the DC componenent is clearly overestimated in the library. 
of the total capture cross section at $k T=25 \mathrm{keV}$, it is then possible to disentangle the direct and the resonant contribution in the astrophysically interesting energy regime. It turns out that the direct capture component is almost negligible, ranging from less than $10 \%$ to less than $1 \%$ between 10 and $100 \mathrm{keV}$. The comparison of the experimental data with the latest release of TENDL indicates that the resonant component is well described, but the direct capture component is overestimated.

\section{ACKNOWLEDGMENTS}

We are very grateful for the excellent support by the entire team of the TRIGA reactor in Mainz. This work was supported by the Helmholtz Young Investigator project, Grant No. VH-NG-327; the BMBF, Project No. 05P12RFFN6; and the Helmholtz International Center for FAIR and HGS-HIRe. K.S. acknowledges support by the DFG (Grant No. SO907/2-1). C.L. acknowledges support from the Austrian Science Fund (FWF), Grant No. J3503.
[1] A. Wallner, M. Bichler, K. Buczak, R. Dressler, L. K. Fifield, D. Schumann, J. H. Sterba, S. G. Tims, G. Wallner, and W. Kutschera, Phys. Rev. Lett. 114, 041101 (2015).

[2] E. A. Samworth, E. K. Warburton, and G. A. P. Engelbertink, Phys. Rev. C 5, 138 (1972).

[3] D. M. Smith, Astrophys. J. 589, L55 (2003).

[4] M. J. Harris, J. Knödlseder, P. Jean, E. Cisana, R. Diehl, G. G. Lichti, J.-P. Roques, S. Schanne, and G. Weidenspointner, Astron. Astrophys. 433, L49 (2005).

[5] F. X. Timmes, S. E. Woosley, D. H. Hartmann, R. D. Hoffman, T. A. Weaver, and F. Matteucci, Astrophys. J. 449, 204 (1995).

[6] C. M. Baglin, Nucl. Data Sheets 95, 215 (2002).

[7] M. Limongi and A. Chieffi, Astrophys. J. 647, 483 (2006).

[8] R. Reifarth, C. Lederer, and F. Käppeler, J. Phys. G 41, 053101 (2014).

[9] E. Uberseder, R. Reifarth, D. Schumann, I. Dillmann, C. D. Pardo, J. Görres, M. Heil, F. Käppeler, J. Marganiec, J. Neuhausen et al., Phys. Rev. Lett. 102, 151101 (2009).

[10] K. Eberhardt and A. Kronenberg, Kerntechnik 65, 5 (2000).

[11] G. Hampel, K. Eberhardt, and N. Trautmann, Atomwirtschaft 5, 326 (2006).

[12] D. Schumann, J. Neuhausen, I. Dillmann, C. Domingo Pardo, F. Käppeler, J. Marganiec, F. Voss, S. Walter, M. Heil, R. Reifarth et al., Nucl. Instrum. Methods Phys. Res., Sect. A 613, 347 (2010).

[13] R. Reifarth, C. Arlandini, M. Heil, F. Käppeler, P. Sedychev, A. Mengoni, M. Herman, T. Rauscher, R. Gallino, and C. Travaglio, Astrophys. J. 582, 1251 (2003).
[14] R. Reifarth and F. Käppeler, Phys. Rev. C 66, 054605 (2002).

[15] J. Apostolakis, techical report, CERN, GEANT Library (1993), http://wwwinfo.cern.ch/asd/geant/.

[16] S. Dababneh, N. Patronis, P. Assimakopoulos, J. Görres, M. Heil, F. Käppeler, D. Karamanis, S. O'Brien, and R. Reifarth, Nucl. Instrum. Methods Phys. Res., Sect. A 517, 230 (2004).

[17] E. Browne and J. K. Tuli, Nucl. Data Sheets 114, 1849 (2013).

[18] N. Nica, Nucl. Data Sheets 111, 525 (2010).

[19] S. K. Basu, G. Mukherjee, and A. A. Sonzogni, Nucl. Data Sheets 111, 2555 (2010).

[20] M. R. Bhat, Nucl. Data Sheets 88, 417 (1999).

[21] G. Rugel, T. Faestermann, K. Knie, G. Korschinek, M. Poutivtsev, D. Schumann, N. Kivel, I. Günther-Leopold, R. Weinreich, and M. Wohlmuther, Phys. Rev. Lett. 103, 072502 (2009).

[22] S. Mughabghab, Atlas of Neutron Resonances, Resonance Parameters and Thermal Cross Sections, $Z=1-100$ (Elsevier, Amsterdam, 2006).

[23] M. Berglund and M. E. Wieser, Pure Appl. Chem. 83, 397 (2011).

[24] A. Koning and D. Rochman, Nucl. Data Sheets 113, 2841 (2012), Special Issue on Nuclear Reaction Data, http://www. sciencedirect.com/science/article/pii/ S0090375212000889.

[25] I. Dillmann, R. Plag, F. Käppeler, and T. Rauscher, EFNUDAT Fast Neutrons-Scientific Workshop on Neutron Measurements, Theory \& Applications (JRC-IRMM, Geel, 2009), Vol. 55, 2009. 